J. Lake Sci.(湖泊科学) , 2016, 28(5): 925-934

DOI 10. 18307/2016. 0501

(C) 2016 by Journal of Lake Sciences

\title{
土壤-作物系统中微囊藻毒素生态毒性的研究进展和展望”
}

\author{
操 庆 $^{1,2}$, 谢丽强 $^{1 \text { ** }}$ \\ (1: 中国科学院南京地理与湖泊研究所湖泊与环境国家重点实验室,南京 210008) \\ (2: 中国科学院大学, 北京 100049)
}

\begin{abstract}
摘 要: 随着蓝藻水华环境问题的日益严重,微囊藻毒素 $(M C s)$ 的污染问题也越来越受到重视. 一些藻毒素污染严重的 湖泊、水库等周边区域的土壤也受到了影响. 用含有藻毒素的地表水及地下水进行灌溉、将蓝藻作为有机肥等措施都会 将 MCs 带人土壤. 一旦 MCs 进人到土壤, 它将会随着降水从地表迁移到土壤深层. 这将会对作物的生长以及土壤生态系 统造成影响, 而且会对人类身体健康造成威胁. 本文介绍了土壤中不同种类的 MCs 对一些粮食作物和蔬菜作物生长的影 响、在作物体内的积累情况, 以及对地下水的污染情况, 概述了 MCs 的致毒机理, 分析了其对土壤生物造成影响的可能 性,并对今后的研究方向进行了展望.
\end{abstract}

关键词: 微囊藻毒素; 土壤; 陆生植物; 地下水;土壤微生物

\section{Progress and prospect of eco-toxicity of microcystins in soil-plant system}

\section{CAO Qing ${ }^{1,2} \&$ XIE Liqiang ${ }^{1 * *}$}

(1: State Key Laboratory of Lake Science and Environment, Nanjing Institute of Geography and Limnology, Chinese Academy of Sciences, Nanjing 210008, P.R. China)

(2: University of Chinese Academy of Sciences, Beijing 100049, P.R.China)

\begin{abstract}
With the increasingly severe environmental problems caused by cyanobacteria blooms, more attentions are paid to the microcystins (MCs) pollution. MCs can be brought into the soils by irrigation with the cyanobacterial blooms polluted water. A lot of cyanobacterial blooms had been taken out of lakes in past several years. Some of the collection water was directly inoculated in soil as organic fertilizer. Once toxic cyanobacterial collections are put into soil, MCs will be released and migrate from the surface to the deeper layers of the soil after precipitation penetration. Therefore, there is a possibility that soils near the lakes and reservoirs might be contaminated by MCs that caused a harmful effect on soil ecosystems and terrestrial plants, including food crop plants, which may correspondingly pose a serious threat to human and animal health. In this paper, effects of MCs on the accumulation, growth and development of terrestrial plants are introduced, as well as the MCs contamination to groundwater involved. We summarized the toxic mechanism of MCs and discussed the possibility of the effect of MCs on soil organism. A future orientation on the soil MCs research is also discussed.
\end{abstract}

Keywords: Microcystins; soil; terrestrial plants; groundwater; soil microorganism

在全球范围内由于湖泊、水库等水体的富营养化而导致的蓝藻水华问题日趋严重. 藻细胞释放出各种 各样有毒的化合物, 其中蓝藻产生的一种单环七肽肝细胞毒性物质微囊藻毒素 (Microcystins, MCs) 量最大, 危害也最为严重. 据报道, 自然界中已经有超过 85 种 MCs 的异构体, 它们化学性质稳定, 耐高温, 不易挥发, 且一般的净水技术都不能有效去除 $\mathrm{MCs}^{[1]}$. 国内外关于 MCs 的研究也越来越多, MCs 能够破坏细胞内的蛋 白磷酸化平衡, 还会导致肝脏血液淤积以及低血容量性休克, 最终导致哺乳动物死亡 ${ }^{[2]}$. 世界卫生组织将饮

* 中国科学院百人计划项目 (Y3BRO11050) 和中国科学院南京地理与湖泊研究所 “一三五” 战略发展规划项目 (NIGLAS2012135015) 联合资助. 2015-11-17 收稿;2016-01-05 收修改稿. 操庆 (1989 ), 男, 博士研究生; E-mail: okcaoqing@163.com.

** 通信作者;E-mail:lqxie@ niglas.ac.cn. 
用水中 MC-LR 的最高浓度暂行标准定位 $1 \mu \mathrm{g} / \mathrm{L}^{[3]}$. MCs 是蓝藻毒素中对安全饮用水生产威胁最大的毒素 种类, 由于其潜在的致癌性, MCs 对人类健康和基础生态过程造成巨大威胁 ${ }^{[4]}$. 虽然 $\mathrm{MCs}$ 是由藻类产生在水 体中, 但由于水体与陆地之间存在各种各样的物质和能量的交换, MCs 通过一些途径也会进人土壤中, 如用 含有藻毒素的水进行灌溉、直接用蓝藻作为肥料施用到田地以及河岸渗滤等措施 ${ }^{[5-8]}$. 土壤中的 MCs 会直接 对种植的陆生植物的生长造成影响, 也会通过在一些陆生植物体内的积累而进人食物链, 还会对地下水造 成污染, 最后, 也会对土壤中的生物造成影响. 因此, 只有正确认识 MCs 进人土壤后造成的危害, 才能更好地 评估 MCs 对土壤环境的影响及其生物安全风险.

\section{1 对陆生植物的影响}

\section{1 对陆生植物生长的影响}

不同的 MCs 变体对于不同的植物有不同的毒性效应 (表 1). Kurki-Helasmo 等 ${ }^{[9]}$ 的研究表明,在浓度为 $5 \mu \mathrm{g} / \mathrm{ml}$ 的 MC-RR 影响下, 白芥的种子萌发受到明显的抑制, 而且在浓度达到 20 40 $\mu \mathrm{g} / \mathrm{ml}$ 时, 其根系的生 长受到明显的抑制. Hamvas 等 ${ }^{[10]}$ 的研究也表明, MC-LR 抑制了芥末的生长, 其鲜物质重、叶片和籽苗主根 的长度都有所减少, 而其 POD 活性有所增加. Pichardo 等 ${ }^{[11]}$ 对几种豆类中抗氧化酶活性受 MCs 影响的研究 中指出, 用 $5.0 \mu \mathrm{g} / \mathrm{L}$ 的 MC-LR 灌溉其籽苗后, 其 POD、GST 和谷胱甘肽还原酶 (GR) 的活性均受到不同程度 的影响. 50 和 $100 \mu \mathrm{g} / \mathrm{L}$ 的 MCs 能够影响亘豆的生长、生节和氮吸收 ${ }^{[12]}$. McElhiney 等 ${ }^{[13]}$ 对马铃薯的研究表 明, MC-LR 能够抑制植株生长 $(\geqslant 5 \mu \mathrm{g} / \mathrm{L})$, 减少根系数量 $(\geqslant 10 \mu \mathrm{g} / \mathrm{L})$, 造成组织细胞坏死和降低叶绿素浓 度 $(\geqslant 50 \mu \mathrm{g} / \mathrm{L})$. 在浓度为 $1.6 \sim 7.7 \mu \mathrm{g} / \mathrm{ml}$ 的 3 种不同 MCs 变体的影响下, 菜豆的光合作用受到明显的抑制.

水稻和甘蓝型油菜在不同浓度 $\mathrm{MCs}(24 \sim 3000 \mu \mathrm{g} / \mathrm{L})$ 的影响下, 其生长发育均有显著性差异 ${ }^{[14]}$. Prieto 等 ${ }^{[15]}$ 的研究发现, MCs 能够影响水稻的生长和氧化应激状态. 高浓度的 MC-LR $(0.5 \sim 4 \mu \mathrm{g} / \mathrm{ml})$ 能够通过抑 制根系的伸长、冠根的形成以及侧根的发生来阻止水稻根系的形态发生 ${ }^{[16]}$. Chen 等 ${ }^{[17]}$ 关于 MCs 对苹果、油 菜和水稻的研究还证实了 MCs 能够引起植物 DNA 的损伤. MCs 对一些人类直接食用的植物的生长率也有 抑制作用, 如西蓝花 (Brassica oleracea ${ }^{[18]}$ 和菠菜 ${ }^{[19]} . \mathrm{MCs}(5.9 \sim 56.4 \mu \mathrm{g} / \mathrm{L}$ ) 菌株对莴亘 (Lactuca sativa) 根系 的生长有明显的抑制作用 ${ }^{[20]}$. 用浓度为 $50 \mathrm{mg} / \mathrm{L}$ 的 MC-RR 处理 $144 \mathrm{~h}$ 后, 烟草 “BY-2” 细胞系 (Nicotiana tabacum L. cv. Bright Yellow 2) 中有活性氧生成, 细胞活性下降 $80 \%{ }^{[21]}$. 用 $5.0 \mu \mathrm{g} / \mathrm{L} \mathrm{MCs}$ 处理过的紫花苜宿 籽苗发芽率和根系生长都受到抑制, 而且出现了明显的氧化应激作用 ${ }^{[22]}$.

总的来说, MCs 能够影响很多陆生植物 (表 1), 造成其产量和品质的下降. 现有的研究还没能够清楚阐 述 MCs 对植物生长影响的机制, 长期低剂量 MCs 对植物生长影响的研究也未见报道, 所以 MCs 对陆生植物 生长的影响还需长期、深人和系统的研究.

\section{2 在陆生植物体内的积累}

国内外也有很多关于 MCs 在陆生植物体内积累的研究 (表 2). Saqrane 等 ${ }^{[32]}$ 研究了用含有 MCs 的水灌 溉硬粒小麦、玉米、踠豆和兵豆后其生理变化. 结果表明, MCs 在不同植物中积累的含量不同, 同种植物的不 同器官中 MCs 积累的含量也不同, 一般是根中积累的 MCs 含量要大于茎和叶. 用浓度为 $4.20 \mu \mathrm{g} / \mathrm{ml}$ 的 MCs 处理踠豆后, 其根、茎和叶中 MCs 含量分别为 190.85 、79.19 和 $156.8 \mu \mathrm{g} / \mathrm{g}$. 小麦根、茎、叶中积累的 MCs 含量 分别为 $16.66 、 1.17$ 和 $15.17 \mu \mathrm{g} / \mathrm{g}$. 当用低浓度 $(0.50 \mu \mathrm{g} / \mathrm{ml})$ 的 MCs 处理这几种作物时, 玉米植株内未检测到 MCs, 其他 3 种植物仍有不同程度的 MCs 积累. Mohamed 等 ${ }^{[33]}$ 用含有 MCs 的水灌溉蔬菜后, 6 种蔬菜根系和 叶片内都检测到 MCs, 且植株体内 MCs 的总量与灌溉水中 MCs 的浓度呈正相关 $(r=0.92)$, 其中夢卜中 MCs 含量最高, 为 $1.2 \mu \mathrm{g} / \mathrm{g}$ ( 鲜重), 卷心菜中 $\mathrm{MCs}$ 含量最低, 为 $0.07 \mu \mathrm{g} / \mathrm{g}$ (鲜重). 除莳萝外, 其他 5 种蔬菜积累 的 MCs 含量都是根系中大于叶片中. 1999 年 Codd 等 ${ }^{[19]}$ 用含有铜绿微囊藻 (Microcystis aeruginosa) (MC-LR 含量为 $3.23 \mathrm{mg} / \mathrm{kg}$ (干重) ) 的水喷灌莴亘, 其叶片基部的 MC-LR 含量为 $0.094 \mathrm{mg} / \mathrm{kg}$ (干重), 叶片末梢为 $0.883 \mathrm{mg} / \mathrm{kg}$ (干重), 叶片中部为 $2.487 \mathrm{mg} / \mathrm{kg}$ (干重). Crush 等 ${ }^{[34]}$ 研究了用含有 MCs 的湖水灌溉对黑麦草、 白三叶、油菜和生菜生长及其 MCs 含量的影响, 茎秆灌溉处理中, 黑麦草和油菜的茎秆中检测不出 MCs, 而 生菜和白三叶中 MCs 含量分别为 0.79 和 $0.20 \mathrm{mg} / \mathrm{kg}$ (干重), 直接灌溉到根部处理中, 根部 MCs 含量最高的 是白三叶, 为 $1.45 \mathrm{mg} / \mathrm{kg}$ (干重), 生菜为 $0.68 \mathrm{mg} / \mathrm{kg}$ (干重), 黑麦草为 $0.20 \mathrm{mg} / \mathrm{kg}$ (干重), 最低的为油菜, 
表 1 微囊藻毒素对陆生植物生长的影响

Tab.1 Effect of microcystins on the growth and development of terrestrial plants

\begin{tabular}{|c|c|c|c|c|}
\hline 植物种类 & MCs 种类 & MCs 浓度/( $\mu \mathrm{g} / \mathrm{L})$ & 影响 & 参考文献 \\
\hline \multirow[t]{6}{*}{ 甘蓝型油菜( Brassica napus) } & $\mathrm{LR}+\mathrm{RR}+\mathrm{YR}$ & $24 \sim 3000$ & $\begin{array}{l}\text { 超氧化物歧化酶 (SOD) 活性降低; 过氧 } \\
\text { 化物酶 (POD) 活性增强 }\end{array}$ & {$[14]$} \\
\hline & & $\geqslant 120$ & 种子萌发和幼苗生长受到抑制 & \\
\hline & & $\geqslant 600$ & 叶片失绿 & \\
\hline & & 3000 & 根尖坏死; 叶片坏死 & \\
\hline & $\mathrm{LR}$ & 0.9 & 蛋白磷酸酶受到抑制 & {$[23]$} \\
\hline & $\mathrm{RR}$ & $\geqslant 100$ & 生长受到抑制 & {$[24]$} \\
\hline \multirow[t]{3}{*}{ 小白菜( Brassica campestris) } & $\mathrm{RR}$ & $\geqslant 100$ & 生长受到抑制 & {$[24]$} \\
\hline & & $\geqslant 600$ & 幼苗根系更短 & \\
\hline & & 3000 & 初生根受到抑制;SOD 活性降低 & \\
\hline 红薯( Ipomoea batatas) & LR & 1988 & $\begin{array}{l}\text { 蛋白磷酸酶受到抑制; 阻碍蔗糖诱导基 } \\
\text { 因的表达 }\end{array}$ & {$[25]$} \\
\hline 家独荇菜 (Lepidium sativum) & $\mathrm{LR}$ & 10 & $\begin{array}{l}\text { 生长受到抑制; 谷胱甘肽颈基转移酶 } \\
\text { (GST) 和脱氧核糖核酸酶活性增强 }\end{array}$ & {$[26]$} \\
\hline \multirow[t]{2}{*}{ 莴苣( Lactuca sativa) } & $\mathrm{LR}+\mathrm{LA}+\mathrm{AR}$ & $5.9 \sim 56.4$ & 根系生长受到抑制 & {$[20]$} \\
\hline & LR & $3000 \sim 6000$ & 芽伸长受到抑制;鲜重减少 & {$[27]$} \\
\hline 苹果( Malus pumila) & $\mathrm{RR}+\mathrm{LR}+\mathrm{YR}$ & $300 \sim 3000$ & $\begin{array}{l}\text { 生长和分芽繁殖受到抑制; POD 和 SOD } \\
\text { 活性增强 }\end{array}$ & {$[28]$} \\
\hline 紫花苜宿 ( Medicago sativa) & LR & 5.0 & 发芽和根系生长受到抑制; 氧化胁迫 & {$[22]$} \\
\hline 烟草( Nicotiana tabacum) & $\mathrm{RR}$ & 50000 & BY-2 细胞系中活性氧积累; 细胞活性下降 & {$[21]$} \\
\hline \multirow[t]{2}{*}{ 水稻 (Oryza sativa) } & $\mathrm{LR}+\mathrm{RR}+\mathrm{YR}$ & $24 \sim 120$ & SOD 活性增高 & {$[14]$} \\
\hline & LR & $500 \sim 4000$ & $\begin{array}{l}\text { 根系的伸长、冠根的形成以及侧根的发 } \\
\text { 生受到抑制 }\end{array}$ & {$[16]$} \\
\hline \multirow[t]{3}{*}{ 菜豆( Phaseolus vulgaris) } & $\mathrm{LR}$ & $\geqslant 9950$ & $\begin{array}{l}\text { 叶绿素含量减少; 光合作用受到抑制; 叶 } \\
\text { 片坏死 }\end{array}$ & {$[29]$} \\
\hline & $\mathrm{LR}$ & 1000 & $\begin{array}{l}\text { 根褐变; 侧根减少; 介质吸收受到抑制; } \\
\text { 叶片坏死 }\end{array}$ & {$[13]$} \\
\hline & $\mathrm{LR}$ & 5.0 & 抗氧化系统改变 & {$[11]$} \\
\hline \multirow[t]{6}{*}{ 白芥 (Sinapis alba) } & $\mathrm{RR}$ & $500 \sim 1000$ & 生长受到抑制 & {$[9]$} \\
\hline & & $\geqslant 5000$ & 茎秆畸形 & \\
\hline & & 10000 & 植物褐变 & \\
\hline & & 20000 & 阻碍根系生成 & \\
\hline & LR & $3500 \sim 30000$ & $\begin{array}{l}\text { 生长受到抑制; 侧根减少; 子叶组织坏 } \\
\text { 死; 叶柄表皮毛缺失; 花青素积累受到抑 } \\
\text { 制;脱氧核糖核酸酶活性增强 }\end{array}$ & {$[10]$} \\
\hline & & $3000 \sim 5000$ & $\begin{array}{l}\text { 生长受到抑制; 子叶中花青素形成受到 } \\
\text { 抑制 }\end{array}$ & {$[30]$} \\
\hline \multirow[t]{2}{*}{ 马铃薯( Solanum tuberosum) } & LR & $10 \sim 100$ & 生长受到抑制 ;根系数量减少 & {$[13]$} \\
\hline & & $50 \sim 5000$ & $\begin{array}{l}\text { 细胞组织坏死; 叶绿素含量减少; 根系发 } \\
\text { 育受到抑制 }\end{array}$ & \\
\hline 菠菜 (Spinacia oleracea) & $\mathrm{LR}$ & 994 & 蔗糖代谢受到抑制 & {$[31]$} \\
\hline 硬粒小麦( Triticum durum) & $\begin{array}{l}R R 、 L R 、 Y R 、 \\
(H 4) 、 Y R 、 W R 、 F R\end{array}$ & 500 & 植株的高度、叶片数量和鲜重都减少 & {$[32]$} \\
\hline 虫豆( Vicia faba) & $\begin{array}{l}\text { DMC-LR、MC- }(\mathrm{H} 4)- \\
\mathrm{YR} 、 L Y 、 C-F R 、 L R\end{array}$ & $50 \sim 100$ & $\begin{array}{l}\text { 抑制种子发芽; 枝干和根系的干重减少; } \\
\text { 结节生长抑制 }\end{array}$ & {$[12]$} \\
\hline 玉米 (Zea mays) & $\begin{array}{l}\text { RR、LR、YR、 } \\
(H 4) Y R 、 W R 、 F R\end{array}$ & 2100 & 叶绿素 $a 、 b$ 含量减少; 光合作用减弱 & {$[32]$} \\
\hline
\end{tabular}


为 $0.12 \mathrm{mg} / \mathrm{kg}$ (干重). 没有直接证据表明植株体内的 MCs 会在根系和茎秆之间迁移. Järvenpää 等 ${ }^{[18]}$ 用含有 1 和 $10 \mu \mathrm{g} / \mathrm{L} \mathrm{MCs}$ 的水来灌溉西兰花和白芥, 在其植株中, 只有根系中能检测到 MC-LR, 分别为 $0.9 \sim 2.4$ 和 $2.5 \sim 2.6 \mu \mathrm{g} / \mathrm{kg}$ (鲜重).

世界卫生组织将饮用水中 MC-LR 的最高含量暂行标准定位为 $1 \mu \mathrm{g} / \mathrm{L}$. 从以往的调查研究来看, 根据正 常人的饮食习惯,农作物可食部分积累的 MCs 含量远远超过人体能够接受的水平. 现有的研究中对于作物 中 MCs 的提取与测定方法不尽相同, 这有可能会导致测定结果之间存在差异性. 鉴于 MCs 在植物 (尤其是 直接食用的蔬菜等作物) 中的积累问题越来越严重, 有必要规范植物体内 MCs 的提取及测定方法, 并对 MCs 污染严重的地区进行植物体内 MCs 积累情况的调查.

\section{2 对地下水的影响}

在全球水资源鿒乏的今天, 地下水是绝大部分人口日常用水的重要来源, 是人类自然资源的重要部分, 工农业生产也离不开地下水资源. 在我国, 以地下水为饮用水源的人口约有 $70 \%$, 农村直接以地下水为饮用 水源的人口超过 $95 \%$, 而全国以地下水灌溉的耕地约有 $40 \%{ }^{[39]}$. MCs 通过各种形式进人土壤后, 对地下水 造成了一定影响.

Chen 等 ${ }^{[7]}$ 对 MCs 进人土壤后对地下水保护的风险评估进行过详细的研究, 以 3 种 MCs 变体 MC-RR、 MC-LR 和 MC-Dha ${ }^{7} \mathrm{LR}$ 为研究对象, 研究其在土壤中的吸收、降解以及移动性. 淋溶实验表明, MC-RR 在淋 出液中的回收率为 $0 \sim 16.7 \%$; MC-LR 为 73.2\% 88.9\% ; MC-Dha ${ }^{7} \mathrm{LR}$ 为 $8.9 \% \sim 73.1 \% .3$ 种 MCs 在土壤中都 有较强的移动性. 地表水中的 MCs 在 4 周内会降解 ${ }^{[40-41]}$. 然而, 由于缺少细菌作用, 地下水中的 MCs 浓度经 过 $100 \mathrm{~d}$ 都没有显著变化 ${ }^{[42]}$. 因此, 在雨季的时候, 将蓝藻水华当做有机肥料施在农田里会导致 MC-LR 等 微囊藻毒素淋溶进人地下水, 造成地下水污染.

Eynard 等 ${ }^{[43]}$ 关于湖水中蓝藻毒素风险的研究中指出, 在蓝藻水华暴发的时候 ( $7-8$ 月) 研究地区的土 壤净化蓝藻毒素的作用有限, 在与地下水相连的水葲站中采集的水样中检测到了相当于 600 和 $1470 \mathrm{ng} / \mathrm{L}$ 的 MC-LR 毒素, 地下水和饮用水仍然受到蓝藻毒素的威胁. Tian 等 ${ }^{[44]}$ 研究了中国淮河流域癌症高发区地表 水和地下水中溶解的 MCs. $51.7 \%$ 的地下水样品中检测出 $\mathrm{MCs}$, 平均浓度为 $0.06 \mu \mathrm{g} / \mathrm{L}$, 最高为 $0.446 \mu \mathrm{g} / \mathrm{L}$. 研究区域地下水中的 MCs 很有可能是因为河流中高浓度的 MCs 的迁移, 越来越多的 MC-RR 和 MC-LR 从河 流中转移到地下水中,地下水中的 MCs 对直接饮用地下水的人们造成很大的潜在健康威胁.

由于河流、湖泊以及池塘附近的渗透作用,包括蓝藻细菌在内的微生物造成的地下水污染时有发生. 降 雨在藻类以及其产物向地下水迁移的过程中也发挥了重要作用. 一旦藻类及藻类孢子进人地下水后, 只要 环境适宜 (如适宜的光照和养分), 它们便会发芽、生长. 由于很多蓝藻都会产生毒素, 所以含有蓝藻的地下 水对水质影响巨大. Mohamed 等 ${ }^{[33]}$ 对阿西尔地区地下井的调查研究表明, 地下井中的浮游植物样品中蓝藻 占主要部分, 而蓝藻中又以颤藻为主. 从地下井采取的浮游植物样品中检测出 $\mathrm{MCs}$ 的最高浓度为 $1100 \mu \mathrm{g} / \mathrm{g}$ (干重). 沙特阿拉伯的阿西亚地区, 在一些不含有能产生 MCs 的藻类及细菌的地下井中也检测出了 $\mathrm{MCs}$, 这说明受污染的地下井水也受到其他水源的渗透影响.

Chen 等 ${ }^{[45]}$ 研究了基于土壤处理机械收集蓝藻水华所带来的风险. 一旦蓝藻水华及含有毒素的水体被 转移到土壤界面, 将不可避免地导致高浓度的 MCs 进人土壤界面, 随后在灌溉水以及雨水等的作用下, MCs 和其他一些毒素则会从土壤表面转移进入地下水, 造成地下水污染. 实验表明, 当土壤中的 MCs 浓度超过 $2.5 \mu \mathrm{g} / \mathrm{L}$ 时, 则有很高的淋溶可能. 实验中地下水样里的 MCs 浓度为 $0.3 \sim 1.3 \mu \mathrm{g} / \mathrm{L}$. 所有样品中的 $\mathrm{MCs}$ 浓 度均超过了促癌作用的限值. 由于地下水一旦被 MCs 污染, 治理起来非常困难, 所以一定要保护好地下 水源.

目前对于 MCs 污染地下水的研究还相对较少, 对于 MCs 污染地区的地下水的调查也不多见, 而用地下 水作为直接饮用水源的人口众多, 因此开展 $\mathrm{MCs}$ 污染地区地下水调查工作刻不容缓, 同时, 应该开展针对地 下水 MCs 污染治理方法的研究. 
表 2 微囊藻毒素在陆生植物体内的积累

Tab.2 The bioaccumulation of microcystins in terrestrial plants

\begin{tabular}{|c|c|c|c|c|}
\hline 植物种类 & MCs 种类、浓度及含量 & 暴露时间 & 植株中 MCs 的积累量 & 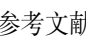 \\
\hline 硬粒小麦 & MC-LR: $0.50 、 1.05$ 和 $4.20 \mathrm{mg} / \mathrm{L}$ & $30 \mathrm{~d}$ & $\begin{array}{l}\text { 根: } 0.18 、 7.6 、 16.66 \mu \mathrm{g} / \mathrm{g} \text { (鲜重) } \\
\text { 茎: ND、ND、1.17 } \mu \mathrm{g} / \mathrm{g} \text { (鲜重) } \\
\text { 叶:ND、ND、15.17 } \mu \mathrm{g} / \mathrm{g} \text { (鲜重) }\end{array}$ & {$[32]$} \\
\hline 玉米 & & & 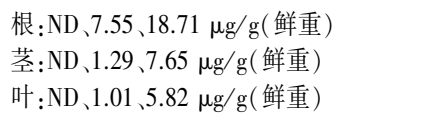 & \\
\hline 踠豆(Pisum sativum) & & & $\begin{array}{l}\text { 根: } 0.24 、 6.23 、 190.85 \mu \mathrm{g} / \mathrm{g} \text { (鲜重) } \\
\text { 茎: ND } 0.88 、 79.19 \mu \mathrm{g} / \mathrm{g} \text { (鲜重) } \\
\text { 叶: } 0.41 、 5.52 、 156.8 \mu \mathrm{g} / \mathrm{g} \text { (鲜重) }\end{array}$ & \\
\hline 兵豆(Lens esculenta cultivars) & & & $\begin{array}{l}\text { 根: } 16 、 24.52 、 162.79 \mu \mathrm{g} / \mathrm{g} \text { (鲜重) } \\
\text { 茎: ND、2.33、36.61 } \mu \mathrm{g} / \mathrm{g} \text { (鲜重) } \\
\text { 叶: } 0.33 、 7 、 98.37 \mu \mathrm{g} / \mathrm{g} \text { (鲜重) }\end{array}$ & \\
\hline $\begin{array}{l}\text { 萝卜 (Raphanus sativus)、芝麻菜 (Eruca } \\
\text { sativ)、生 菜 ( Lactuca sativa)、莳 萝 } \\
\text { (Anethum graveolens)、欧芹 }(\text { Petroselinum } \\
\text { crispum)、卷心菜 }(\text { Brassica oleracea })\end{array}$ & MCs: $0.3 \sim 1.8 \mu \mathrm{g} / \mathrm{L}$ & 整个生长季 & $\begin{array}{l}\text { 在灌溉水浓度最高为 } 1.8 \mu \mathrm{g} / \mathrm{L} \text { 时, 萝卜 } \\
\text { 约为 } 1.2 \mu \mathrm{g} / \mathrm{g} \text { 、芝麻菜约为 } 0.85 \mu \mathrm{g} / \mathrm{g} \text { 、 } \\
\text { 生菜约为 } 0.45 \mu \mathrm{g} / \mathrm{g} \text { 、莳萝约为 } 0.87 \\
\mu \mathrm{g} / \mathrm{g} \text { 、欧芹约为 } 0.87 \mu \mathrm{g} / \mathrm{g} \text { 、卷心菜约 } \\
\text { 为 } 0.87 \mu \mathrm{g} / \mathrm{g} \text { (鲜重) }\end{array}$ & {$[33]$} \\
\hline $\begin{array}{l}\text { 黑麦草 ( Lolium perenne)、白三叶 ( Tri- } \\
\text { folium repens)、甘蓝型油菜 (Brassica } \\
\text { napa)、生菜 }\end{array}$ & MCs: $1.6 \mathrm{mg} / \mathrm{L}$ & 整个生长季 & $\begin{array}{l}\text { 茎秆灌溉处理: 黑麦草和油菜茎秆未 } \\
\text { 检出,白三叶和生菜分别为 } 0.20 \mathrm{mg} / \\
\mathrm{kg} \text { 和 } 0.79 \mathrm{mg} / \mathrm{kg} \text { (干重); } \\
\text { 根部灌溉处理:白三叶为 } 1.45 \mathrm{mg} / \mathrm{kg} \text { 、 } \\
\text { 生菜为 } 0.68 \mathrm{mg} / \mathrm{kg} \text { 、黑麦草为 } 0.20 \\
\mathrm{mg} / \mathrm{kg} \text { 、油菜为 } 0.12 \mathrm{mg} / \mathrm{kg} \text { (干重) }\end{array}$ & {$[34]$} \\
\hline 西兰花 & $\begin{array}{l}\text { MCs: 浓度 } 0 、 1 、 10 \mu \mathrm{g} / \mathrm{L}, \text { 总量 } 0 、 \\
2.6 、 26 \mu \mathrm{g}\end{array}$ & $20 \mathrm{~d}$ & $\begin{array}{l}\text { 只有根部检测出 MC-LR:0.9 } 2.4 \mu \mathrm{g} / \\
\mathrm{kg} \text { (鲜重) }\end{array}$ & {$[18]$} \\
\hline 白芥 & $\begin{array}{l}\text { MCs: 浓度 } 0 、 1 、 10 \mu \mathrm{g} / \mathrm{L} \text {, 总量 } 0 、 2.3 \text { 、 } \\
23 \mu \mathrm{g}\end{array}$ & $19 \mathrm{~d}$ & $\begin{array}{l}\text { 只有根部检测出 MC-LR:2.5 } 2.6 \mu \mathrm{g} / \\
\mathrm{g} \text { (鲜重) }\end{array}$ & \\
\hline 青菜 (Brassica chinensis var. chinensis) & $\begin{array}{l}\text { MC-LR: } 0.008 、 0.04 、 0.08 、 0.4 、 0.8 \text { 、 } \\
4 \mathrm{mg} / \mathrm{L}\end{array}$ & $7 \mathrm{~d}$ & $\begin{array}{l}\text { MC-LR 含量: } 40.2 、 103.7 、 156.2 、 214.9 、 \\
533.5 、 1609.4 \mu \mathrm{g} / \mathrm{kg} \text { (鲜重) } \\
\text { MC-RR 含量: } 12.9 、 19.1 、 26.2 、 33.6 、 44.0 、 \\
104.7 \mu \mathrm{g} / \mathrm{kg} \text { (鲜重) }\end{array}$ & [35] \\
\hline 甘蓝型油菜 & $\begin{array}{l}\text { MC-RR : } 0 、 10 、 50 、 100 、 500 、 1000 、 \\
5000 \mu \mathrm{g} / \mathrm{L}\end{array}$ & $7 \mathrm{~d}$ & $\begin{array}{l}\text { MC-LR 含量: } 48.74 、 67.76 、 78.43 、 \\
109.93 、 215.84 、 546.94 \mu \mathrm{g} / \mathrm{kg} \text { (鲜重) } \\
\text { MC-RR 含量: } 11.28 、 16.81 、 28.32 、 \\
33.88 、 47.82 、 84.62 \mu \mathrm{g} / \mathrm{kg} \text { (鲜重) }\end{array}$ & {$[36]$} \\
\hline 甘蓝型油菜 & $\begin{array}{l}\text { 蓝藻粗提物, MC-LR 浓度: } 0.024 \sim \\
3.000 \mathrm{mg} / \mathrm{L}\end{array}$ & $10 \mathrm{~d}$ & $651 \mu \mathrm{g} / \mathrm{kg}$ (鲜重) & {$[14]$} \\
\hline 水稻 & & & $5.4 \mu \mathrm{g} / \mathrm{kg}$ (鲜重) & \\
\hline 白三叶 & MC-RR : $0.05 、 0.1 、 0.5 、 1 、 5 \mathrm{mg} / \mathrm{L}$ & $7 \mathrm{~d}$ & $\begin{array}{l}\text { MC-RR 含量: } 82 、 126 、 157 、 196 、 582 \\
\mu \mathrm{g} / \mathrm{kg} \text { (鲜重) }\end{array}$ & {$[37]$} \\
\hline 生菜 & $\begin{array}{l}\text { Microcystis aeruginosa 浸 提物 中 } \\
\text { MC-LR 含量为 } 3.23 \mathrm{mg} / \mathrm{kg} \text { (干重) }\end{array}$ & 整个生长季 & $\begin{array}{l}\text { MC-LR 在叶片中含量为: 基部 } 0.094 \\
\mathrm{mg} / \mathrm{kg} \text { (干重); 中部 } 2.487 \mathrm{mg} / \mathrm{kg} \text { (干 } \\
\text { 重);末梢 } 0.883 \mathrm{mg} / \mathrm{kg} \text { (干重) }\end{array}$ & {$[19]$} \\
\hline 黑麦草 & MC-LR: $0.04 、 0.08 、 0.4 、 0.8 、 4 \mathrm{mg} / \mathrm{L}$ & $7 \mathrm{~d}$ & $\begin{array}{l}\text { MC-LR 含量: } 0 、 0.3 、 3.56 、 6.37 、 39.48 \\
\mathrm{mg} / \mathrm{kg} \text { (干重) }\end{array}$ & {$[38]$} \\
\hline 菜豆 & MC-LR: $1.12 \mathrm{mg} / \mathrm{L}$ & $18 \mathrm{~d}$ & 整株浸提液 MC-LR 含量为 $19.1 \mathrm{mg} / \mathrm{kg}$ & {$[13]$} \\
\hline
\end{tabular}




\section{3 对土壤生物的影响}

MCs 的致毒机理目前主要有 3 种解释: 对蛋白磷酸酶的抑制作用、对细胞的氧化胁迫和内质网应激. MCs 能够抑制苏氨酸和丝氨酸蛋白磷酸酶 ( protein phosphatases, PPs) 1 以及 $2 \mathrm{~A}$ 的活性, 而蛋白磷酸酶在一 系列的生理生化反应中有重要作用,所以当其活性被抑制后,会破坏细胞内的蛋白磷酸化平衡,损害细 胞 ${ }^{[46]}$; 当 MCs 进人生物体后, 使得细胞内活性氧簇 (reactive oxygen species, ROS) 急剧产生, 细胞内抗氧化 系统受到影响, SOD、GSH、过氧化氢酶( CAT) 等都会改变, 大量的 ROS 还会导致膜脂过氧化、对 DNA 造成损 伤、与细胞膜和生物大分子物质发生作用, 引起细胞和组织损伤 ${ }^{[47-48]}$; 关于内质网应激的研究目前还很少, 可能是由 MCs 促使折叠的蛋白质在内质网内腔的积累而导致的 ${ }^{[49]}$. 但对于像蓝藻这样的原核生物微生物 来说, $\mathrm{MCs}$ 的致毒机理是否相同还不确定, 还没有研究证实原核生物中存在和真核生物类似的蛋白磷酸酶 基因,因此在原核生物中, MCs 抑制蛋白磷酸酶不一定会对其产生影响,但 MCs 对细胞的氧化胁迫同样会影 响原核微生物, 使其细胞内的生理生化特性发生改变. 同样, 对于作为真核生物的土壤动物来说, MCs 势必 也会对它们的生长产生影响.

MCs 对细菌和真菌的影响机制目前还没有明确的认识. 关于 MCs 对水体中细菌和真菌的影响作用国内 外已经有所研究, Valdor 等的研究发现 ${ }^{[50]}$, 用微囊藻粗提物和纯的 MC-RR 处理大肠杆菌 ( Streptoverticillium sp.) 和链需菌 (Escherichia coli), 它们的生长均受到不同程度的抑制作用, 但不致死. 而 Singh 等 ${ }^{[51]}$ 对绿藻、 蓝藻细菌、异养细菌和真菌的研究发现, 微囊藻的粗提取物和纯的 MCs 只对绿藻和蓝藻的生长有抑制作用, 而对其他没有影响. 刘玲莉等 ${ }^{[52]}$ 研究发现, 微囊藻的提取液对其胶鞘上伴生的细菌假单胞菌 (Pseudomonas sp.) 的生长不但没有抑制, 反而有促进作用, 并可取代酵母膏提供其生长所需的生长因子等营养物质. Dixon 等 ${ }^{[53]}$ 的研究指出, $2.5 \mathrm{mg} / \mathrm{L}$ 的 MCs 会促进溶酶菌进人大肠杆菌. 国内也有关于 MCs 能够改变细胞膜透性的 研究, 杨翠云 ${ }^{[54]}$ 也发现纯微囊藻毒素 MC-RR 对大肠杆菌和枯草芽孢杆菌的生长及细胞活性具有短暂的抑 制应, 并且能够促进细胞膜对溶酶菌的通透性, 促进细胞内可溶性糖和蛋白的外渗; MC-RR 对大肠杆菌和枯 草芽孢杆菌的氧化胁迫具有一定的时间和剂量效应. 由于不同的细菌和真菌结构和抗性不同, MCs 对其影 响作用效果也不尽相同. 从这些研究中可以看出, MCs 能够改变细胞膜的渗透性而使大分子化合物进入细 胞, 这将会对生态系统中细菌和真菌的数量造成一定影响, 并在细菌和真菌种类竞争中具有重要的生态学 功能 ${ }^{[55-57]}$.

目前关于 MCs 对土壤微生物直接影响的报道尚不多见. 2015 年 Corbel 等 $^{[58]}$ 对土壤-植物系统中含有 $\mathrm{MCs}$ 的蓝藻粗提物的植物毒性和生态毒性的可能性进行了评估. 用含有 MCs (浓度相当于 $0.1 \mathrm{mg} / \mathrm{L} \mathrm{MC-LR}$ ) 的水灌溉土壤 $14 \mathrm{~d}$ 后, 土壤中的芳基硫酸酥酶、磷酸酶、腿酶和 $\beta$-D-葡萄糖苷酶活性受 MCs 影响均不显著, 但低浓度的 MCs 显著增加了土壤中的硝化势, 最大约为空白处理的 5 倍. 不同 MCs 浓度处理的土壤中细菌 和古菌的 $16 S r R N A$ 基因丰度没有显著变化, 氨氧化古菌 $a m o A$ 基因丰度也没有显著变化, 然而不同浓度 $\mathrm{MCs}$ 处理后的土壤中氨氧化细菌 $a m o A$ 基因丰度均显著增加. 但因为实验中的土壤种植了作物, 所以土壤中 这些微生物的变化有可能是由于植物根系活动影响造成的. 因此, 关于纯 MCs 对土壤微生物的影响还需进 一步的研究.

国内外尚无关于 $\mathrm{MCs}$ 对土壤动物影响的报道, 土壤动物在生态系统中举足轻重,一方面积极同化各种 有用物质以建造其自身,另一方面又将其排泄物归还到环境中不断改造环境. MCs 进人土壤后必然会对生 活在其中的土壤动物产生影响. MCs 对土壤动物的毒性作用还亟待研究, 需要开展的研究包括 MCs 对土壤 动物的急性毒性(如存活、摄食)、慢性毒性(如生长、繁殖)、对其生理生化的影响 (如蛋白酶、磷酸酶活性)、 对其行为及分布的影响等.

\section{4 展望}

由于全球气候变化等原因, 水体富营养化问题在水生生态系统, 尤其是湖泊和水库中越来越严重, 蓝藻 水华在咸水和淡水水体中频发, 且范围越来越广. MCs 存在于全世界很多国家和地区的天然水体中. 随着 MCs 污染范围的逐渐扩大, 越来越多的研究者渐渐地将目光从湖泊、水库等水体上转移到了土壤中来. 土壤 
是个复杂的自然体, 与人类生产、生活息息相关. 进人土壤的 MCs 将会对土壤相关的各种生物产生影响,包 括陆生植物甚至人类. 因此, 在 MCs 对人类健康影响的风险评估中, 以及在评价 MCs 污染严重的湖泊及水 库周边的农业生产质量和土壤质量过程中, 全面了解 MCs 进人土壤后对植物、地下水以及土壤生物的影响 以及其作用机制十分迫切和必要.

关于进人土壤生态系统中 MCs 的研究开展得较早, 但大部分研究集中在地上部分, 对陆生植物影响的 研究相对较多, 而对土壤生物方面的研究则较为缺乏. 在已有的研究中, 已经认识到 MCs 经过各种途径进人 土壤后, 会对种植在土壤上的作物生长造成影响, 也会在一些作物体内积累, 这样不但会影响作物的产量和 品质, 而且会对以此作物为食的人类的健康造成威胁; 由于在土壤中的移动性以及雨水等的作用, MCs 往往 会对地下水造成污染, 这对直接饮用地下水的人群的身体健康也造成了极大威胁, 而且用 MCs 污染的地下 水进行灌溉, 又会对作物产生影响; 土壤动物在土壤生态系统中担负着消费者和分解者的多重角色, 在土壤 的形成、熟化以及在生态系统的能量转化和物质循环中都起着重要作用. 土壤微生物是土壤生物活性的重 要组分, 是土壤性质的一个重要方面, 直接或间接地参与了几乎所有的土壤过程. MCs 进人土壤后对土壤生 物造成影响, 长此以往势必会影响土壤的质量. 但尚有一些问题亟需解决, 如目前还没有蓝藻水华污染严重 的一些湖泊、水库周边的土壤、植物和地下水中 MCs 的调查数据, 无法估计 MCs 对人类健康影响的风险值; MCs 对植物生长影响的作用机制尚不明确, MCs 的含量与作物生长受影响程度以及作物中的积累量的剂量 效应关系也不明确, 缺乏长时间低剂量的 MCs 对作物生长影响的研究; 现有的研究都集中在粮食作物以及 蔬菜作物上, 很少有关于经济作物 (如果树等) 对 MCs 积累的研究; 缺乏关于 MCs 对土壤生物影响的研究, 现在关于 MCs 对水生生物影响的研究有很多, 但国内外尚无 MCs 对土壤中一些大型动物 (如环节动物、节 肢动物和软体动物等) 影响的研究, 对土壤微生物的影响作用也鲜有研究. 因此, 目前还无法评估 MCs 对土 壤质量的影响作用.

针对目前的研究现状以及上述存在的问题,建议今后的研究工作重点可以放在以下几个方面:

1) 全面调查 MCs 污染区域的土壤、植物以及地下水中的 MCs 种类、含量以及分布. 为准确评估 MCs 对 人类健康影响的风险值奠定基础.

2) 加强 MCs 对植物生长影响的作用机制的研究; 阐明 MCs 与植物的剂量效应关系, 调查研究 MCs 在污 染区域种植较多较广的经济作物中的积累作用以及对作物品质的影响. 从而有效控制 MCs 在土壤-植物系 统中的迁移和生态风险.

3) 全面开展 MCs 对土壤生物影响的研究, 主要是对土壤微生物的研究, 包括微囊藻毒素对土壤酶活性、 土壤微生物群落结构以及土壤微生物功能的影响, 以及对其他土壤动物生长、多样性及分布格局的影响, 为 MCs 污染区域的土壤质量评估提供依据.

\section{5 参考文献}

[ 1 ] Rivasseau C, Martins S, Hennion MC. Determination of some physicochemical parameters of microcystins ( cyanobacterial toxins ) and trace level analysis in environmental samples using liquid chromatography. Journal of Chromatography, 1998, $799(1 / 2): 155-169$.

[ 2 ] Dziga D, Wasylewski M, Wladyka B et al. Microbial degradation of microcystins. Chemical Research in Toxicology, 2013, 26(6) : 841-852.

[ 3 ] Kirsten C, Hanne K. Toxic cyanobacteria in water. A guide to their public health consequences, monitoring, and management. Limnology \& Oceanography, 2000, 45(5) : 1212.

[ 4 ] Rastogi RP, Sinha RP, Incharoensakdi A. The cyanotoxin-microcystins: current overview.Reviews in Environmental Science \& Bio/technology, 2014, 13(2): 215-249.

[ 5 ] Morris RJ, Williams DE, Luu HA et al. The adsorption of microcystin-LR by natural clay particles. Toxicon, 2000,38 (2) : 303-308.

[ 6 ] Miller MJ, Critchley MM, Hutson J et al. The adsorption of cyanobacterial hepatotoxins from water onto soil during batch experiments. Water Research, 2001, 35(6) : 1461-1468.

[ 7 ] Chen W, Song LR, Gan NQ et al. Sorption, degradation and mobility of microcystins in Chinese agriculture soils: Risk as- 
sessment for groundwater protection. Environmental Pollution, 2006, 144(3) : 752-758.

[ 8 ] Rapala J, Sivonen K, Lyra C et al. Variation of microcystins, cyanobacterial hepatotoxins, in Anabaena spp. as a function of growth stimuli. Applied and Environmental Microbiology, 1997, 63 (6) : 2206-2212.

[ 9 ] Kurki-Helasmo K, Meriluoto J. Microcystin uptake inhibits growth and protein phosphatase activity in mustard (Sinapsis alba L. ) seedlings. Toxicon, 1998, 36(12) : 1921-1926.

[10] Hamvas MM, Mathe C, Molnar E et al. Microcystin-LR alters the growth, anthocyanin content and single-stranded DNase enzyme activities in Sinapsis alba L. seedlings. Aquatic Toxicology, 2003, 62(1) : 1-9.

[11] Pichardo S, Pflugmacher S. Study of the antioxidant response of several bean variants to irrigation with water containing MC-LR and cyanobacterial crude extract. Environmental Toxicology, 2011, 26(3) : 300-306.

[12] Lahrouni M, Oufdou K, Faghire M et al. Cyanobacterial extracts containing microcystins affect the growth, nodulation process and nitrogen uptake of faba bean (Vicia faba L., Fabaceae). Ecotoxicology, 2012, 21(3) : 681-687.

[13] McElhiney J, Lawton LA, Leifert C. Investigations into the inhibitory effects of microcystins on plant growth, and the toxicity of plant tissues following exposure. Toxicon, 2001, 39(9) : 1411-1420.

[14] Chen J, Song L, Dai J et al. Effects of microcystins on the growth and the activity of superoxide dismutase and peroxidase of rape (Brassica napus L.) and rice (Oryza sativa L.). Toxicon, 2004, 43(4) : 393-400.

[15] Prieto A, Campos A, Camea'n A et al. Effects on growth and oxidative stress status of rice plants (Oryza sativa) exposed to two extracts of toxin-producing cyanobacteria (Aphanizomenon ovalisporum and Microcystis aeruginosa). Ecotoxicology \& Environmental Safety, 2011, 74(7) : 1973-1980.

[16] Chen J, Han FX, Wang F et al. Accumulation and phytotoxicity of microcystin-LR in rice (Oryza sativa). Ecotoxicology \& Environmental Safety, 2012, 76(2) : 193-199.

[17] Chen JZ, Ye JY, Zhang HY et al. Freshwater toxic cyanobacteria induced DNA damage in apple (Malus pumila), rape (Brassica napus) and rice (Oryza sativa). Journal of Hazardous Materials, 2011, 190(s 1/2/3) : 240-244.

[18 ] Järvenpää S, Lundberg-Niinistö C, Spoof L et al. Effects of microcystins on broccoli and mustard, and analysis of accumulated toxin by liquid chromatography-mass spectrometry. Toxicon, 2007, 49(6) : 865-874.

[19] Codd GA, Metcalf JS, Beattie KA. Retention of Microcystis aeruginosa and microcystin by salad lettuce (Lactuca sativa) after spray irrigation with water containing cyanobacteria. Toxicon, 1999, 37(8) : 1181-1185.

[20] Pereira S, Saker ML, Vale M et al. Comparison of sensitivity of grasses (Lolium perenne L. and Festuca rubra L.) and lettuce (Lactuca sativa L.) exposed to water contaminated with microcystins. Bulletin of Environmental Contamination \& Toxicology, 2009, 83(1): 81-84.

[21] Yin L, Huang J, Huang W et al. Microcystin-RR-induced accumulation of reactive oxygen species and alteration of antioxidant systems in tobacco BY-2 cells. Toxicon, 2005, 46(5) : 507-512.

[22] Pflugmacher S, Jung K, Lundvall L et al. Effects of cyanobacterial toxins and cyanobacterial cell-free crude extract on germination of alfalfa (Medicago sativa) and induction of oxidative stress. Environmental Toxicology \& Chemistry, 2006, 25 (9) : 2381-2387.

[23] MacKintosh C, Beattie KA, Klumpp S et al. Cyanobacterial microcystin-LR is a potent and specific inhibitor of protein phosphatases 1 and 2A from both mammals and higher plants. Febs Letters, 1990, 264(2): 187-192.

[24] Liu BB, Gong Y, Xiao BD et al. A laboratory study on risk assessment of microcystin-RR in cropland. Journal of Environmental Management, 2008, 86(3): 566-574.

[25] Takeda S, Mano S, Ohto M et al. Inhibitors of protein phosphatases 1 and 2A block the sugar-inducible gene expression in plants. Plant Physiology, 1994, 106(2) : 567-574.

[26] Gehringer MM, Kewada V, Coates N et al. The use of Lepidium sativum in a plant bioassay system for the detection of microcystin-LR. Toxicon, 2003, 41(7): 871-876.

[27] Wang Z, Xiao B, Song L et al. Effects of microcystin-LR, linear alkylbenzene sulfonate and their mixture on lettuce ( Lactuca sativa L.) seeds and seedlings. Ecotoxicology, 2011, 20(4) : 803-814.

[28] Chen J, Dai J, Zhang H et al. Bioaccumulation of microcystin and its oxidative stress in the apple (Malus pumila). Ecotoxicology, 2010, 19(4) : 796-803.

[29] Abe T, Lawson T, Weyers DB et al. Microcystin-LR inhibits photosynthesis of Phaseolus vulgaris primary leaves: implications for current spray irrigation practice. New Phytologist, 1996, 133(4) : 651-658. 
[30] Hamvas MM, Máthé C, Vasas G et al. Cylindrospermopsin and microcystin-LR alter the growth, development and peroxidase enzyme activity of white mustard (Sinapis alba L.) seedlings, a comparative analysis. Acta Biologica Hungarica, 2010, 61 ( Suppl 1) : 35-48.

[31] Siegl G, MacKintosh C, Stitt M. Sucrose-phosphate synthase is dephosphorylated by protein phosphatase 2A in spinach leaves.Febs Letters, 1990, 270(1/2): 198-202.

[32] Saqrane S, Ouahid Y, El Ghazali I et al. Physiological changes in Triticum durum, Zea mays, Pisum sativum and Lens esculenta cultivars, caused by irrigation with water contaminated with microcystins: a laboratory experimental approach. Toxicon, 2009, 53(7/8) : 786-796.

[33] Mohamed ZA, Shehri AMA. Microcystins in groundwater wells and their accumulation in vegetable plants irrigated with contaminated waters in Saudi Arabia. Journal of Hazardous Materials, 2009, 172(1) : 310-315.

[34] Crush JR, Briggs LR, Sprosen JM et al. Effect of irrigation with lake water containing microcystins on microcystin content and growth of ryegrass, clover, rape, and lettuce. Environmental Toxicology, 2008, 23(2) : 246-252.

[35] Li Huiming, Xue Yanfeng, Yi Neng et al. Effects of microcystins on the growth of Chinese cabbage (Brassica chinensis L.) and its accumulation in seedlings. Jiangsu Journal of Agricultural Sciences, 2009, 25(3) : 680-684( in Chinese with English abstract). [ 李慧明, 薛延丰, 易能等. 藻毒素对青菜生长的影响及其在幼苗体内的积累. 江苏农业学报, 2009, 25(3) : 680-684.]

[36] Yi Neng, Xue Yanfeng, Li Huiming et al. Effects of Microcystins on the growth and activities of antioxidant enzymes in rape seedling. Acta Botanica Boreali-Occidentalia Sinica, 2009, 29(6) : 1207-1213(in Chinese with English abstract). [易能, 薛延丰, 李慧明等. 微囊藻毒素对油菜幼苗生长及抗氧化酶活性的影响. 西北植物学报, 2009, 29(6): 1207-1213. ]

[37] Xue Yanfeng, Li Huiming, Yi Neng et al. Effects of MC-RR on the seed germination and characteristics of physiology and biochemistry in Trifolium repens seedlings. Acta Prataculturae Sinica, 2009, 18(6) : 180-185(in Chinese with English abstract). [ 薛延丰, 李慧明, 易能等. 微囊藻毒素 (MC-RR) 对白三叶种子萌发及幼苗生理生化特性影响. 草业学报, $2009, \mathbf{1 8}(6)$ : 180-185.]

[38] Geng Zhiming, Gu Yingying, Wang Peng. Effects of microcystin on growth and development of pakchoi cabbage and tomato and its accumulation in them. Acta Agriculturae Jiangxi, 2011, 23(9): 21-24( in Chinese with English abstract). [ 耿志 明, 顾迎迎, 王澎. 微囊藻毒素对小白菜、番茄生长发育影响及其在它们体内积累的研究. 江西农业学报, 2011, $\mathbf{2 3}(9): 21-24$.]

[39] Yi Guoxun, Li Zhenshan eds. Groundwater pollution and prevention. Beijing: China Environmental Science Press, 2005 (in Chinese).[尹国勋, 李振山. 地下水污染与防治. 北京: 中国环境科学出版社, 2005.]

[40] Ishii H, Nishijima M, Abe T. Characterization of degradation process of cyanobacterial hepatotoxins by a gram-negative aerobic bacterium. Water Research, 2004, 38(11) : 2667-2676.

[41] Lahti K, Rapala J, Färdig M et al. Persistence of cyanobacterial hepatotoxin, microcystin-LR in particulate material and dissolved in lake water. Water Research, 1997, 31(5): 1005-1012.

[42] Holst T, Jørgensen NOG, Jørgensen C et al. Degradation of microcystin in sediments at oxic and anoxic, denitrifying conditions. Water Research, 2003, 37(19) : 4748-4760.

[43] Eynard F, Mez K, Walther JL. Risk of cyanobacterial toxins in Riga waters (Latvia). Water Research, 2000, 34( 11) : 2979-2988.

[44] Tian DJ, Zheng WW, Wei X et al. Dissolved microcystins in surface and ground waters in regions with high cancer incidence in the Huai River Basin of China. Chemosphere, 2013, 91(7) : 1064-1071.

[45] Chen W, Jia YL, Li EH et al. Soil-based treatments of mechanically collected cyanobacterial blooms from Lake Taihu: Efficiencies and potential risks. Environmental Science \& Technology, 2012, 46(24) : 13370-13376.

[46] MacKintosh C, Beattie KA, Klumpp S et al. Cyanobacterial microcystin-LR is a potent and specific inhibitor of protein phosphatases 1 and 2A from both mammals and higher plants. FEBS Letters, 1990, 264(2) : 187-192.

[47] Li XY, Liu YD, Song LR et al. Responses of antioxidant systems in the hepatocytes of common carp (Cyprinus carpio L.) to the toxicity of microcystin-LR. Toxicon, 2003, 42(1) : 85-89.

[48] Pflugmacher S. Promotion of oxidative stress in the aquatic macrophyte Ceratophyllum demersum during biotransformation of the cyanobacterial toxinmicrocystin-LR. Aquatic Toxicology, 2004, 70(3) : 169-178. 
[49] Rutkowski DT, Kaufman RJ. A trip to the ER: coping with stress.Trends in Cell Biology, 2004, 14(1) : 20-28.

[50] Valdor R, Aboal M. Effects of living cyanobacteria, cyanobacterial extracts and pure microcystins on growth and ultrastructure of microalgae and bacteria. Toxicon, 2007, 49 (6) : 769-779.

[51] Singh DP, Tyagi MB, Kumar A et al. Antialgal activity of a hepatotoxin-producing cyanobacterium, Microcystis aeruginosa. World Journal of Microbiology and Biotechnology, 2001, 17(1) : 15-22.

[52] Liu Lingli, Gu Yufei, Luo Yu et al. On the growth and phosphorous metabolism of bacterium isolated from Microcystis aeruginosu in Taihu Lake. J Lake Sci, 2000, 12(4) : 373-378(in Chinese with English abstract). DOI: 10.18307/2000. 0412. [ 刘玲莉, 顾宇飞, 罗屿等. 一株自太湖微囊藻上分离到的细菌的生长及磷代谢. 湖泊科学, 2000, 12(4): 373-378.]

[53] Dixon RA, Al-Nazawi M, Alderson G. Permeabilising effects of sub-inhibitory concentrations of microcystin on the growth of Escherichia coli. Fems Microbiology Letters, 2004, 230(2) : 167-170.

[54] Yang Cuiyun. Studies on the ecophysiological effects of microcystins against microbes[Dissertation]. Wuhan: Institute of Hydrobiology, CAS, 2007 (in Chinese with English abstract). [ 杨翠云. 微囊藻毒素对微生物的生理生态学效应 [ 学位 论文]. 武汉: 中国科学院水生生物研究所, 2007.]

[55] Aboal M, Puig MA, Ríos H et al. Relationship between macroinvertebrate diversity and toxicity of Cyanophyceae (Cyanobacteria) in some streams from Eastern Spain. Dublin: Congress of International Association of Theoretical \& Applied Limnology, 2000, 27 (1): 1-5.

[56] Aboal M, Puig MA, Mateo P et al. Implications of cyanophyte toxicity on biological monitoring of calcareous streams in north-east Spain. Journal of Applied Phycology, 2001, 14(1) : 49-56.

[57] Aboal M, Puig MA, Asencio AD. Production of microcystins in calcareous Mediterranean streams: The Alharabe River, Segura River basin in south-east Spain. Journal of Applied Phycology, 2005, 17(3) : 231-243.

[58] Corbel S, Mougin C, Martin-Laurent F et al. Evaluation of phytotoxicity and ecotoxicity potentials of a cyanobacterial extract containing microcystins under realistic environmental concentrations and in a soil-plant system. Chemosphere, 2015, 128: $332-340$. 